\title{
Effect of KRAS exon 2 mutations on antitumor activity of afatinib and gefitinib
}

Sebastian Gamba ${ }^{\mathrm{a}, \star}$, Peter Camaj ${ }^{\mathrm{c}, \star}$, Volker Heinemann ${ }^{\mathrm{a}}$, Rüdiger P. Laubender ${ }^{b, d, e}$, Yan Wang ${ }^{c}$, Yue Zhao ${ }^{c}$, Sebastian Stintzing ${ }^{a}$, Clemens Giessen ${ }^{\mathrm{a}}$, Stefan Boeck ${ }^{\mathrm{a}}$, Christoph Haertl ${ }^{\mathrm{a}}$, Christiane J. Bruns ${ }^{\mathrm{c}, \mathrm{d}, \mathrm{e}}$ and Dominik P. Modest ${ }^{\mathrm{a}}$

The aim of this study was to investigate the impact of different KRAS mutations on the inhibitory potential of afatinib and gefitinib in SW48 colorectal cancer cells. The influence of afatinib/gefitinib on cell viability and cell cycle was evaluated in isogenic SW48 KRAS wild-type/mutant cells. Protein levels of phosphorylated/total EGFR, HER-2, HER-3, ERK, and AKT were compared between treated/ untreated samples using western blotting. The activity of both afatinib and gefitinib was the lowest in KRAS G12C/G12S/G12D and the highest in G13D/G12A mutant subtypes. A $50 \%$ decrease in cell viability was achieved at concentrations of 3.0-7.7 $\mu \mathrm{mol} / \mathrm{I}$ for afatinib and 5.4-19.5 $\mu \mathrm{mol} / \mathrm{I}$ for gefitinib. The effect of both drugs on apoptosis appeared to be stronger than their influence on proliferation and was generally less pronounced in mutant cells than in wild-type cells. The average number of apoptotic cells after treatment with afatinib was 2.6 times as high as the corresponding value following treatment with gefitinib $(P<0.01)$. Levels of pEGFR, pHER-2, pERK, and pAKT were reduced more extensively by afatinib than by gefitinib $(P<0.001)$. Some KRAS mutations (G12C/G12S/G12D) appear to weaken the activity of afatinib and gefitinib

\section{Introduction}

In recent years, therapeutic considerations in metastatic colorectal cancer (mCRC) have been influenced considerably by the advent of monoclonal antibodies (mAbs) targeting the epidermal growth factor receptor (EGFR), an oncogene frequently expressed in various solid tumors, triggering incessant proliferation through a number of interrelated pathways [1-4]. Cetuximab and panitumumab, used either alone or in combination with chemotherapeutic agents, have been shown to improve the outcome of mCRC patients [5-9]. Soon after these agents were introduced into clinical practice, retrospective studies reported a significant correlation between Kirsten rat sarcoma viral oncogene homolog (KRAS) mutational status and therapeutic efficacy, proposing that alterations of this gene, which encodes for a membrane-associated guanosine triphosphatase that influences downstream signaling, might account for inherent resistance to EGFR-targeting mAbs [10-16].

Mutations of the KRAS gene most frequently occur in codons 12 and 13 of exon 2: depending on the specific amino acid exchange involved, they are termed G12A, whereas others seem to increase sensitivity to treatment (G13D/G12A) compared with the parental clone (KRAS wild-type). In SW48 colorectal cancer cells, afatinib seems to be more potent than gefitinib because of its superior efficacy in inhibiting both EGFR and HER-2, suppressing signaling along both MEK/ERK and PI3K/AKT pathways to a greater extent. Anti-Cancer Drugs 26:371-378 Copyright (C) 2015 Wolters Kluwer Health, Inc. All rights reserved.

Anti-Cancer Drugs 2015, 26:371-378

Keywords: afatinib, colorectal cancer, gefitinib, KRAS, tyrosine kinase inhibitors

aDepartment of Medicine III, ' Institute of Medical Informatics, Biometry and Epidemiology, University Hospital Grosshadern, Munich, 'Department of Surgery, University Hospital Magdeburg, Magdeburg, ${ }^{\mathrm{d}}$ German Cancer Consortium (DKTK) and ${ }^{e}$ German Cancer Research Center (DKFZ), Heidelberg, Germany

Correspondence to Dominik P. Modest, MD, Department of Medicine III, University Hospital Grosshadern, Marchioninistrasse 15, 81377 Munich, Germany Tel: + 49897095 3135; fax: + 49897095 5256;

e-mail: dominik.modest@med.uni-muenchen.de

*Sebastian Gamba and Peter Camaj contributed equally to the writing of this article.

Received 15 October 2014 Revised form accepted 15 November 2014

G12C, G12D, G13D, G12R, G12S, and G12V [17]. These mutations activate several intracellular pathways, causing resistance against EGFR-targeting mAbs [18,19]. As a consequence, treatment with cetuximab and panitumumab has been restricted to patients bearing KRAS wild-type tumors, which account for $\sim 60 \%$ of all mCRCs $[17,20]$. Interestingly, as described by De Roock et al. [21], mutations of the KRAS gene are not entirely homogeneous. Some KRAS mutants (G13D in particular) may still respond to treatment with cetuximab/panitumumab, suggesting a varying degree of resistance in $K R A S$ variants [22]. These findings indicate the need for further discrimination of $K R A S$ subtypes, with a particular focus on the identification of those KRAS mutants showing sensitivity to promising drugs currently withheld from respective patients.

Similar to mAbs, small molecule tyrosine kinase inhibitors (TKIs) such as afatinib or gefitinib were also developed to interfere with the above-mentioned structures. Gefitinib acts as a reversible inhibitor of the EGFR tyrosine kinase domain and has proven to be effective in a fraction of patients with non-small-cell lung carcinoma 
[23-26]. By contrast, afatinib irreversibly interferes with an entire set of proteins belonging to the human epidermal growth factor receptor (HER) family of receptor tyrosine kinases, including EGFR and HER-2 [27]. Afatinib is being evaluated in various clinical trials concerning non-small-cell lung carcinoma, metastatic breast cancer, and advanced pancreatic cancer [28-30]. Homodimerization or heterodimerization among members of the HER family is an essential and early step in signal transduction following EGFR ligand binding [1,31, 32]. Although HER-2 has been shown to be the preferred binding partner for other HER receptors, HER-3, although capable of binding ligands, appears to be lacking intrinsic tyrosine kinase activity, thus requiring further downstream signaling to be induced by its respective partner [33,34].

The means by which mutations in the KRAS gene render tumor cell proliferation (at least partly) independent of EGFR stimulation might affect the efficacy of TKIs in a similar manner as observed with mAbs: direct activation of mitogen/extracellular signal-regulated kinase (MEK)/ extracellular-signal-regulated kinase (ERK) and phosphatidylinositide 3-kinase (PI3K)/protein kinase B (AKT) pathways by KRAS is possibly causing TKIs to become ineffectual in $K R A S$ mutant tumors [35-40]. At this point, however, it is not entirely conclusive to what extent other members of the HER family are involved in this matter and what influence different mutations within the KRAS gene truly have on the expression of different EGFRrelated receptors and their susceptibility to treatment. This in-vitro study aims to characterize the varying effect of afatinib/gefitinib on isogenic colorectal cancer cell lines harboring the seven most frequent mutations in the KRAS gene and to decipher the respective contribution of EGFR, HER-2, HER-3, ERK, and AK'T to the degree of sensitivity observed in those cells.

\section{Materials and methods \\ Cell lines}

All experiments were conducted using the human colon adenocarcinoma cell line SW48 (obtained from Horizon Discovery, Cambridge, UK). Apart from the mother cell line expressing wild-type $K R A S$, seven other cell lines harboring the most frequent mutations located in codons 12 and 13 of exon 2 of the KRAS gene (G12A/G12C/G12D/G13D/G12R/ G12S/G12V) were available for the purpose of this investigation. Horizon Discovery engineered these KRAS mutant cell lines using a recombinant adeno-associated viral vector [41-43].

\section{KRAS-testing/KRAS-quantification}

To confirm the KRAS mutation status of all acquired cell lines, detailed analysis was subsequently carried out by a German laboratory licensed for $K R A S$-testing (Department of Pathology, University of Munich, Munich, Germany). Specific detection of KRAS mutations was performed using
Qiagen PyroMark Gold (Qiagen, Venlo, Netherlands) reagents in combination with a Q24 pyrosequencing device [44]. All the cell lines examined were shown to express comparable amounts of KRAS protein.

\section{Cell culture}

Cells were maintained in McCoy's 5A medium (Life Technologies, Carlsbad, California, USA) supplemented with $100 \mathrm{ml} / \mathrm{l}$ fetal bovine serum (Biochrom, Berlin, Germany), $100 \mathrm{U} / \mathrm{ml}$ penicillin, and $100 \mu \mathrm{g} / \mathrm{ml}$ streptomycin (PAN Biotech, Aidenbach, Germany). They were incubated at $37^{\circ} \mathrm{C}$ in an atmosphere of $50 \mathrm{ml} / 1 \mathrm{CO}_{2}$ and $950 \mathrm{ml} / \mathrm{l}$ air. Medium was changed every 3 days and cells were subcultured regularly once confluency of about $70 \%$ was reached.

\section{Drugs}

Afatinib was kindly provided by Boehringer Ingelheim (Ingelheim, Germany). Gefitinib was purchased from LC Laboratories (Woburn, Massachusetts, USA).

\section{Colorimetric cell viability assay}

Cell Counting Kit-8 (CCK-8; Dojindo Molecular Technologies, Gaithersburg, Maryland, USA) was used for colorimetric assessment of cell viability. We began by seeding 3000 cells/well in 96-well tissue culture plates. After $24 \mathrm{~h}$ of incubation at $37^{\circ} \mathrm{C}$, adherent cells were exposed to increasing concentrations of afatinib/gefitinib. Following another $48 \mathrm{~h}$ of incubation, CCK- 8 solution was added to each well according to the manufacturer's recommendations. Cell viability was evaluated using an ELISA plate reader, measuring optical density at a wavelength of $450 \mathrm{~nm}$. The mean $\mathrm{IC}_{50}$ values were calculated after conducting three separate and independent experiments for each cell line and therapeutic setup.

\section{Quantification of proliferating and apoptotic cells using flow cytometry}

Nonconfluent cells were seeded at 75000 cells/well in sixwell tissue culture plates and incubated at $37^{\circ} \mathrm{C}$. Afatinib/ gefitinib was added after $24 \mathrm{~h}$ using the $\mathrm{IC}_{50}$ value determined previously for each respective drug in SW48 $K R A S$ wild-type cells. After an additional $48 \mathrm{~h}$ of incubation, cells were collected and suspended in Nicoletti buffer $(1 \mathrm{mg} / \mathrm{ml}$ sodium citrate at $\mathrm{pH} 7.4,1 \mathrm{mg} / \mathrm{ml}$ Triton $\mathrm{X}-100$, and $50 \mu \mathrm{g} / \mathrm{ml}$ propidium iodide). Nuclear DNA content was subsequently measured using a fluorescenceactivated cell sorter (Becton Dickinson, Heidelberg, Germany). Mean values were calculated from the results of three separate and independent experiments.

\section{Western blot analysis of protein expression}

Cells were treated with afatinib/gefitinib once confluency of about $70 \%$ was reached. As described above, we used $\mathrm{IC}_{50}$ values evaluated previously for $K R A S$ wild-type cells. After a period of $48 \mathrm{~h}$, cells were washed with ice-cold PBS and resuspended in ice-cold complete lysis-M 
buffer (Roche, Mannheim, Germany) supplemented with $5 \mathrm{mmol} / \mathrm{l}$ sodium vanadate (Sigma-Aldrich, St. Louis, Missouri, USA), yielding a final concentration of $10^{7}-10^{8}$ cells/ml. Equal amounts of protein were resolved by SDSPAGE and blotted to polyvinylidene difluoride membranes (Merck Millipore, Billerica, Massachusetts, USA). Proteins of interest were detected using quantum dots (Invitrogen, Karlsruhe, Germany) as well as specific antibodies against phosphorylated/total EGFR, HER-2, HER-3, ERK, and AKT (Cell Signaling Technology, Danvers, Massachusetts, USA). $\beta$-Actin served as a loading control. All antibodies were used according to the manufacturer's instructions.

\section{Statistical analysis}

Data were summarized using appropriate measures of location and spread. A two-way analysis of variance with a factor indicating the type of treatment (afatinib and gefitinib), a factor indicating the cell lines (WT, G12A, G12C, G12D, G13D, G12R, G12S, and G12V), and with the interaction of both factors was carried out. To compare pairwise mean differences, the $P$-values from Tukey's honest significant difference method was used to control the family-wise type I error rate. All tests were two-sided and adjusted $P$-values less than 0.05 were considered statistically significant. The statistical software R (version 2.13.1; R Foundation for Statistical Computing, Vienna, Austria) was used for all calculations.

\section{Results}

\section{Colorimetric cell viability assay}

Compared with $K R A S$ wild-type cells, G13D and G12A mutant cells were more sensitive to gefitinib; all other mutant subtypes were less susceptible to this drug. Except for one case (G12A), these differences were statistically significant $(P<0.01)$. A similar pattern was observed following treatment with afatinib: G13D and G12A mutant cells seemed to be more sensitive compared with KRAS wild-type cells, whereas the other mutant subtypes appeared to be less responsive to afatinib than KRAS wild-type cells. However, the differences found for afatinib were not statistically significant. Overall, a $50 \%$ decrease in cell viability was achieved at concentrations of 3.0-7.7 $\mu \mathrm{mol} / \mathrm{l}$ for afatinib and 5.4-19.5 $\mu \mathrm{mol} / \mathrm{l}$ for gefitinib (Fig. 1).

\section{Quantification of proliferating and apoptotic cells}

Cell cycle analysis was carried out in KRAS wild-type/ mutant cells following $48 \mathrm{~h}$ of treatment, comparing the amount of apoptotic/proliferating cells in treated/ untreated samples (Fig. 2a-c). The influence of gefitinib on apoptosis appeared to be greater in KRAS wild-type cells than in KRAS mutant cell lines. Although treatment resulted in higher rates of apoptotic cells in some mutant variants, compared with the average fraction of apoptotic cells in treated KRAS mutant cells, treatment resulted in 1.7 times as many apoptotic cells in $K R A S$ wild-type cells. Similar to gefitinib, afatinib had a larger impact on
Fig. 1

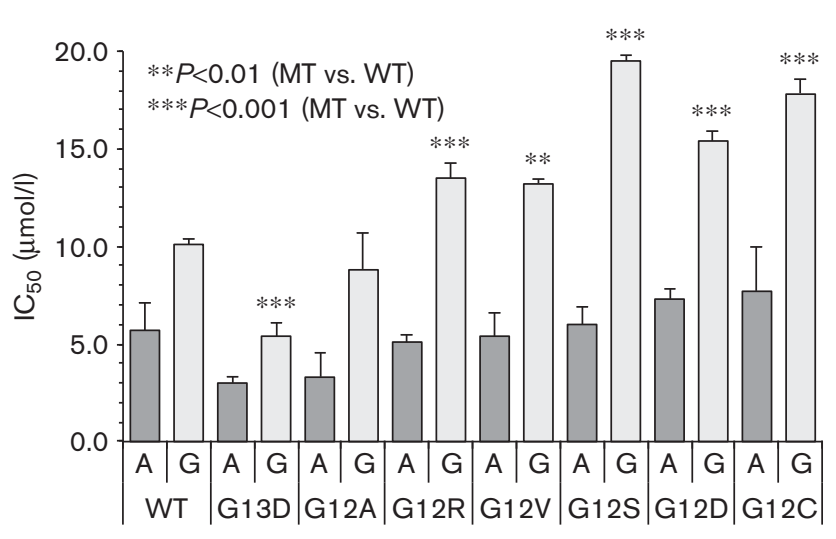

Colorimetric cell viability assay following treatment with afatinib $(A)$ or gefitinib (G). SW48 Kirsten rat sarcoma viral oncogene homolog (KRAS) wild-type cells (WT) were taken as a reference and compared with mutant cells (MT) treated with the same agent.

apoptosis in KRAS wild-type cells than in KRAS mutant cells. In $\mathrm{G} 12 \mathrm{C} / \mathrm{G} 13 \mathrm{D} / \mathrm{G} 12 \mathrm{~S}$ mutant cells treated with afatinib, the fraction of apoptotic cells was 4.8-6.9 times as high as in untreated samples. By contrast, afatinib (like gefitinib) did not increase the number of apoptotic cells in G12A/G12V mutant cells. Differences between KRAS variants in terms of apoptosis did not reach significance. Generally, afatinib seemed to produce a considerably higher amount of apoptotic cells than gefitinib across all cell lines (on average, the number of apoptotic cells after treatment with afatinib was 2.6 times as high as the corresponding value following treatment with gefitinib; $P<0.01)$.

In most cell lines, cell proliferation decreased following treatment with gefitinib. The number of proliferating cells was particularly reduced in G12C/G12D/G13D mutant cells, being merely $0.6-0.7$ times as high as in untreated samples. Cell proliferation was also inhibited by afatinib, with G12C/G12D/G13D mutant cells also being most affected in this respect (0.2-0.4 times as many proliferating cells as in untreated samples). Consistent with its effect on apoptosis, afatinib appeared to have a stronger impact on proliferation than gefitinib. However, differences in cell proliferation were not found to be statistically significant.

\section{Evaluation of EGFR/pEGFR, HER-2/pHER-2, and HER-3/pHER-3 protein levels}

Owing to the fact that afatinib/gefitinib exert effects by inhibiting different members of the HER family, we aimed to evaluate protein levels of total EGFR, HER-2, and HER-3 before/after treatment (Fig. 3a-d). The expression of these receptor proteins appeared to be rather similar among treated/untreated KRAS wild-type/ mutant cell lines. On examining two different sites of 
Fig. 2
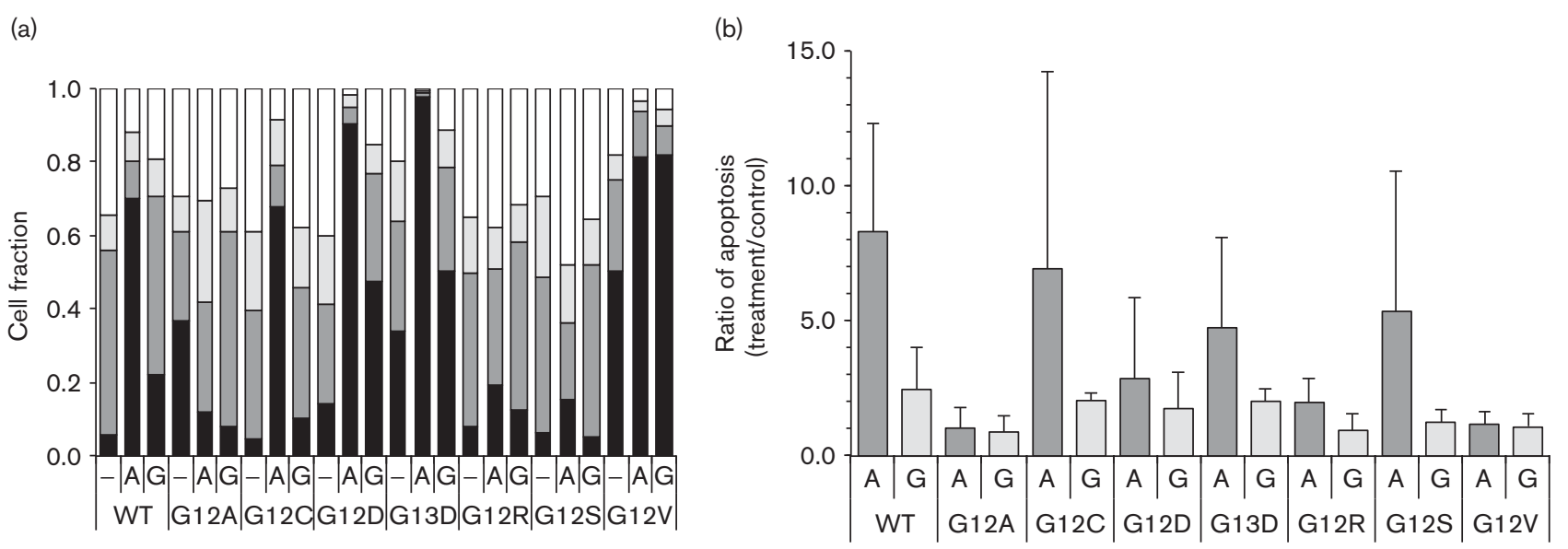

$\square$ M $\square$ S $\square$ G0G1 Apoptosis

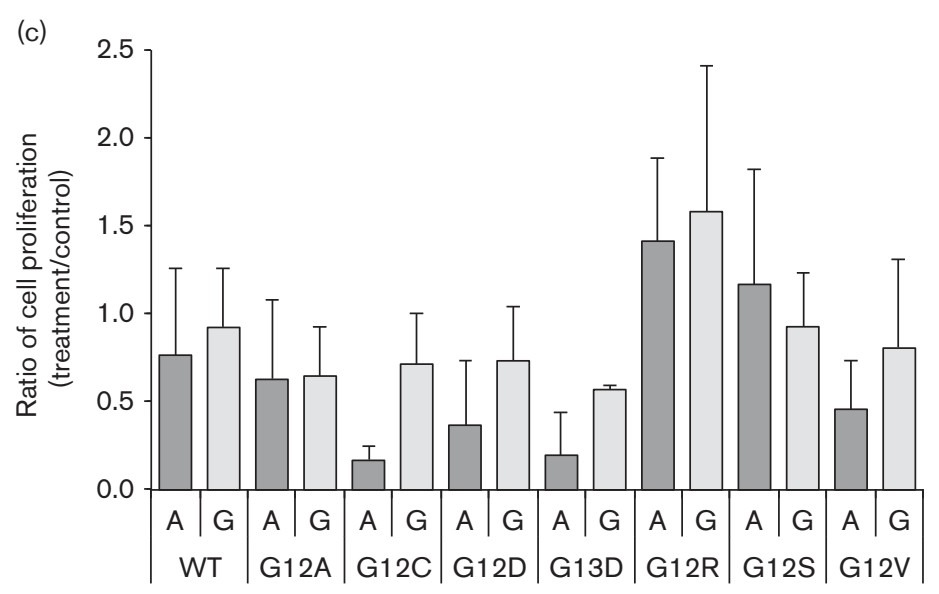

Cell cycle analysis following treatment with afatinib (A) or gefitinib (G). Exemplary FACS result (a). Ratio of apoptotic and proliferating (mitotic) cells in treated samples to the corresponding values in untreated samples (b, c). Values more than 1 indicate an increase in the respective cell fraction in treated samples relative to untreated samples; a decrease is indicated by values less than 1. Mutant cells (MT) were compared with Kirsten rat sarcoma viral oncogene homolog (KRAS) wild-type cells (WT) treated with the same drug.

phosphorylation ('Tyr845/Tyr992), we found that levels of phosphorylated EGFR were affected by treatment with afatinib/gefitinib. In cells treated with gefitinib, Tyr992 phosphorylation was reduced on average by $49 \%$ compared with untreated samples; Tyr845 phosphorylation was reduced by $2 \%$. The decrease in pEGFR was relatively pronounced in $\mathrm{G} 13 \mathrm{D} / \mathrm{G} 12 \mathrm{~S} / \mathrm{G} 12 \mathrm{~V}$ mutant cells and only moderate in G12A/G12C mutant subtypes. On average, afatinib reduced $\mathrm{Tyr} 992$ phosphorylation by $74 \%$ and Tyr845 phosphorylation by 56\%. Again, G13D/G12S/G12V mutant cells were more responsive than G12A/G12C mutant subtypes in this respect. Across all cell lines, afatinib was shown to suppress EGFR activation more effectively than gefitinib $(P<0.001)$. Upon treatment with gefitinib, the expression of pHER-2 remained close to pretherapeutic levels in G12C/G12D mutant cells (decreasing by 7\%) and in $K R A S$ wild-type/G13D mutant cells (increasing by 2-7\%). G12A/G12R/G12V mutant subtypes seemed to be affected to a greater extent, with levels of pHER-2 decreasing by $30-48 \%$. By contrast, treatment with afatinib resulted in a decrease in pHER-2 levels in all except two cell lines (G12C/G13D). KRAS wild-type as well as $\mathrm{G} 12 \mathrm{~A} / \mathrm{G} 12 \mathrm{~V}$ mutant cells were most susceptible to the inhibition of HER-2 phosphorylation by afatinib (pHER-2 levels were reduced by 51-59\%). Overall, afatinib could suppress HER-2 activation to a greater extent than gefitinib: on average, the levels of pHER-2 decreased by 34 and $16 \%$, respectively $(P<0.01)$. The levels of phosphorylated HER-3 generally did not seem to be affected much by either drug.

\section{Evaluation of ERK/pERK and AKT/pAKT protein levels}

We further investigated the expression of several proteins mediating downstream signaling along MEK/ERK and PI3K/AKT pathways, comparing the effect of afatinib/ gefitinib on KRAS wild-type/mutant cells (Fig. 3a, e, f). Once again, we found similar amounts of total ERK/AKT 


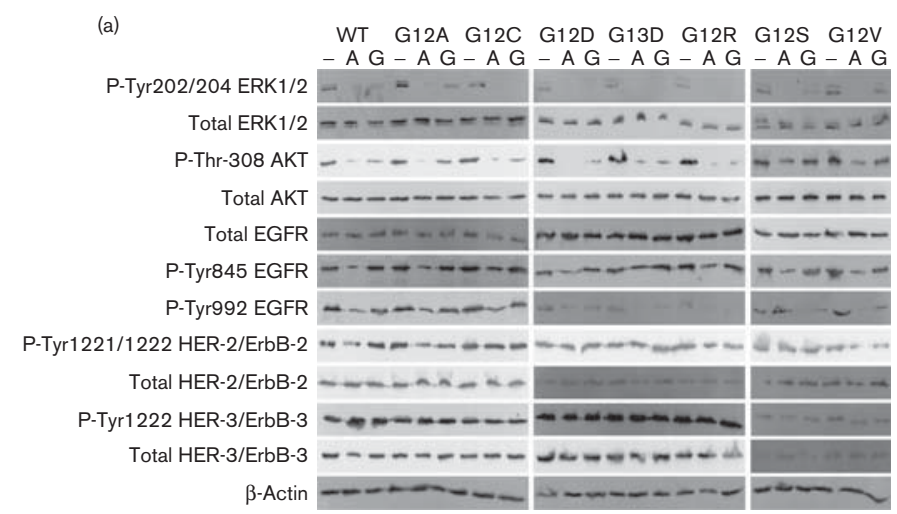

(c)

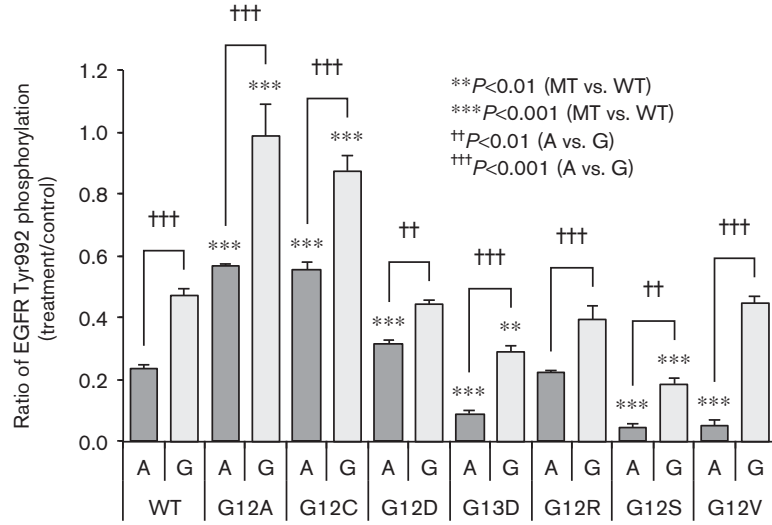

(e)

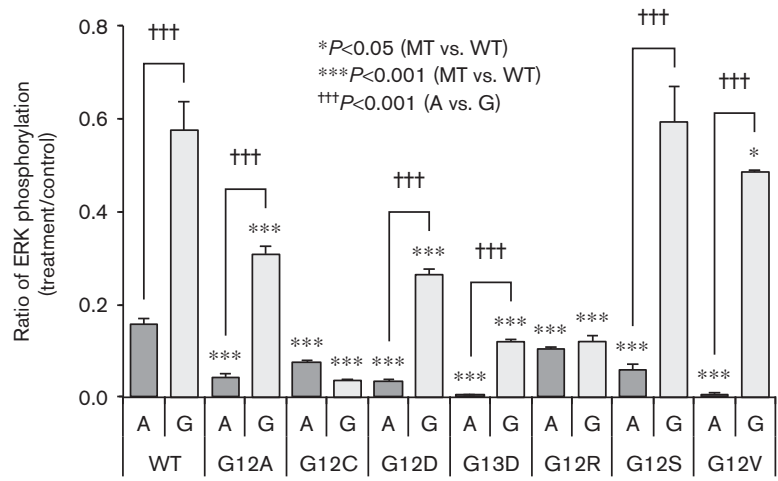

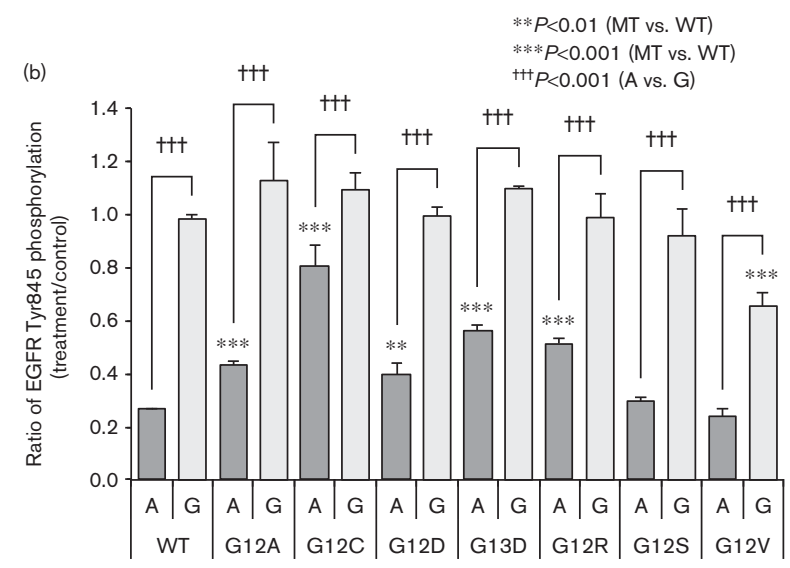

$* P<0.05$ (MT vs. WT) $* * P<0.01$ (MT vs. WT) $* * * P<0.001$ (MT vs. WT)

(d)
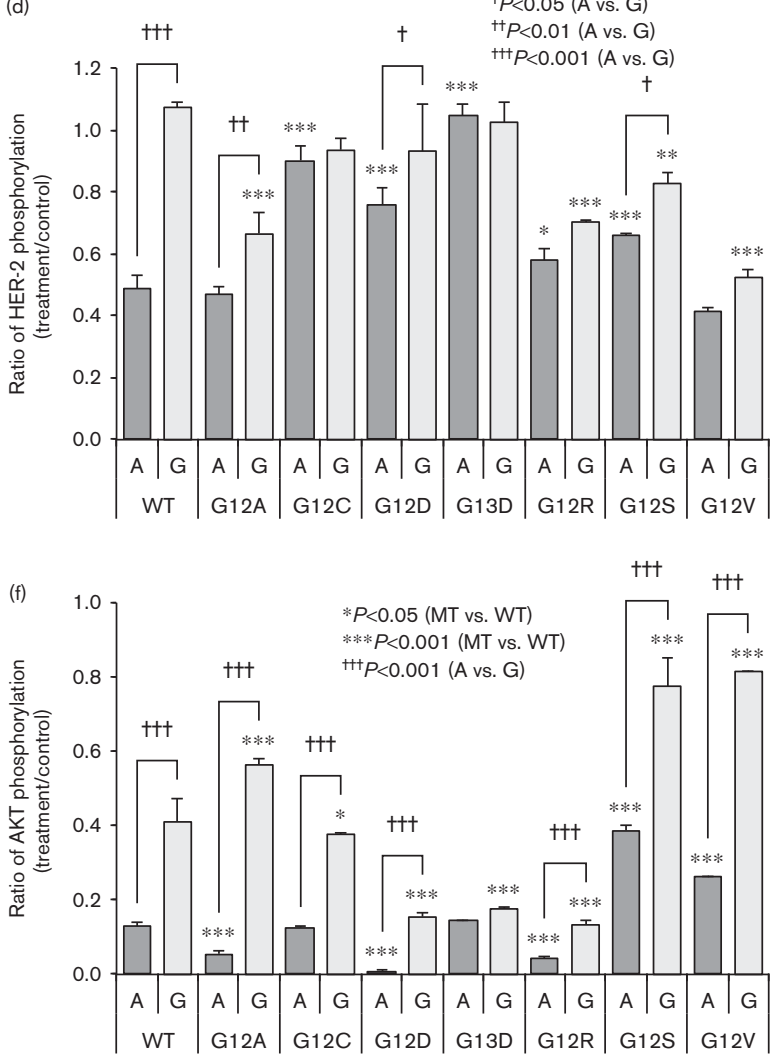

Evaluation of protein levels without $(-)$ and with treatment with afatinib (A) or gefitinib (G). Western blot (a). Relative phosphorylation of the target proteins was quantified by densitometry $(\mathrm{b}-\mathrm{f})$. Mutant subtypes (MT) were compared with Kirsten rat sarcoma viral oncogene homolog (KRAS) wildtype cells (WT) of the same treatment group. AKT, protein kinase B; EGFR, epidermal growth factor receptor; ERK, extracellular-signal-regulated kinase; HER-2, human epidermal growth factor receptor 2.

across all cell lines. Although the levels of pERK were also mostly similar in untreated KRAS mutant/wild-type cells, treatment with gefitinib was shown to elicit strong inhibitory effects on this pathway, leading to a $41-51 \%$ decrease of pERK in KRAS wild-type as well as G12S/G12V mutant cells; a more thorough inhibition was observed in
G12C/G13D/G12R mutant subtypes (88-96\%). In contrast, afatinib reduced levels of pERK the least in KRAS wild-type cells (84\%) and the most in G13D/G12V mutant cells $(>99 \%)$. Our results indicate afatinib to generally have a stronger inhibitory influence on pERK than gefitinib (on average, the levels of pERK were reduced by 94 and 
$69 \%$, respectively; $P<0.001$ ). Levels of phosphorylated AKT were largely similar among untreated KRAS wildtype/mutant cell lines. Treatment with gefitinib resulted in a slight decrease in pAKT levels in G12S/G12V (18-22\%) and a more pronounced reduction in G12A/G12D/G12R mutant cells (85-87\%). Afatinib was shown to produce an even sharper decrease in pAKT levels across most cell lines: phosphorylation decreased the least in G12S/G12V (61-74\%) and the most in G12A/G12D/G12R mutant subtypes (94-99\%). Overall, the levels of pAKT were reduced more extensively by afatinib $(86 \%)$ than by gefitinib $(57 \%)(P<0.001)$.

\section{Discussion}

$K R A S$-dependent efficacy of therapeutic agents targeting proteins of the EGFR pathway has been described in several reports, and retrospective subgroup analyses have shown the poor benefit of such drugs in patients bearing $K R A S$ mutant mCRC tumors [10-16]. In addition, many reports agree that some $K R A S$ mutant variants might still rely on growth stimulation originating from members of the HER family or may be less resistance activating, making them equally or even more sensitive to treatment than wild-type cells $[22,45]$.

Our model of investigation is based on isogenic cell lines showing microsatellite instability. Although this setting of cells bearing heterozygous knock-in mutations is highly artificial, it allowed us to consider the impact of $K R A S$ mutations in an isolated manner, with a single base exchange being the only distinctive feature causing disparities in therapeutic outcome and little interference to be expected from other sources. The consequences of these mutations might be far more complex in vivo, most probably involving several different mechanisms of action that could not be explored in this setting. The scope of our investigation and our findings is further limited by the fact that SW48 colorectal cancer cells do not seem to form metastatic lesions in vivo [46]. It is also important to mention that effects that are specific to the cell line that we used may have possibly influenced our results.

Apart from KRAS wild-type cells, we selected the seven most common mutations and aimed to compare the various effects of afatinib and gefitinib on cell viability, the cell cycle, and levels of regulatory proteins. Although colorimetric cell viability assays provided somewhat coherent information on the degree of sensitivity to afatinib/gefitinib in the examined KRAS wild-type/ mutant cell lines (similar reactions to either treatment), cell cycle analysis and differences between protein levels among KRAS mutant subtypes seemed less conclusive. Certain cell lines that were relatively irresponsive to treatment in cell viability assays (e.g. G12C) and expected to show a comparable behavior in cell cycle analysis turned out to be rather sensitive to the induction of apoptosis and the inhibition of proliferation by both afatinib and gefitinib. In general, we found most cell lines to be more susceptible to the induction of apoptosis than to the inhibition of proliferation.

Afatinib and gefitinib belong to the same class of antineoplastic drugs, and yet our findings suggest that afatinib, acting as an irreversible ErbB family blocker, has a stronger inhibitory effect on EGFR and HER-2 phosphorylation than gefitinib in most of the cell lines examined. As shown previously for cetuximab [47], activation of HER-2 might be a mechanism of resistance possibly attenuating the therapeutic effect of EGFRinhibiting agents. Afatinib may appear to be more effective because of its mechanism of blocking multiple receptor subtypes of the HER family, thus also having a greater inhibitory influence on those receptors that might act as compensatory structures in those cells that are less responsive to gefitinib [48].

Both ERK and AKT are intermediate members of the EGFR signaling cascade and directly/implicitly subordinate to changes in KRAS activation [49]. ERK phosphorylation has been shown to be largely reliant on EGF stimulation, and intrinsic levels of pERK before/ after such stimulation were observed to depend on the mutational status of KRAS [50]. It has also been argued that in KRAS mutant colorectal cancers, KRAS acts as a principal regulator of signaling along the MEK/ERK pathway, but not along the PI3K/AKT pathway (as the latter is considered to require stimulation by receptor tyrosine kinases) [51]. This implies that TKIs would only serve to inhibit AKT in this setting, and that additional agents targeting MEK (or, in fact, other regulatory proteins along the same pathway) would be needed for sufficient therapeutic success. In this investigation, however, KRAS wild-type/mutant cells were found to be highly susceptible to the inhibitory effect of afatinib and gefitinib on both ERK and AKT, indicating that even in $K R A S$ mutant tumors, members of the HER family still retain regulatory control over these pathways.

Although afatinib was generally shown to silence receptor activation more effectively, several KRAS mutant subtypes that were shown to be fairly insensitive to gefitinib (G12D/G12S/G12C) also proved to be relatively irresponsive to treatment with afatinib, suggesting that some cell lines have such little dependency on HER signaling that even combined inhibition of more than one type of receptor only influences cell survival to a marginal extent. Some of these KRAS mutant subtypes are frequently found in clinical samples and appear to be associated with poor outcome when treated with cetuximab-based regimens (esp. G12C); yet, it remains difficult to determine whether these mutations lead to significant resistance against TKIs in vivo [16,44,52]. Consistent with clinical observations [21], we further found that SW48 G13D mutant cells are not only comparatively sensitive to treatment with TKIs $\left(\mathrm{IC}_{50}\right.$ values are lower than those 
evaluated for $K R A S$ wild-type cells), but that afatinib and gefitinib strongly induce apoptosis and inhibit proliferation in this particular KRAS variant as well.

We conclude that the therapeutic impact of both afatinib and gefitinib seems to depend on the KRAS mutation status of tumor cells, with some mutant subtypes responding better (G13D) and others responding worse (G12C/G12S/G12D) to these drugs than wild-type cells. This investigation further showed afatinib to possibly be more potent than gefitinib in certain respects; its efficacy might be a consequence of its strong influence on both EGFR and HER-2. Further preclinical and clinical research on the use of TKIs in solid tumors is warranted, and subgroup analyses of clinical trials involving the use of TKIs on patients carrying KRAS wild-type/mutant tumors will hopefully provide greater insight into the exact behavior of these therapeutic agents.

\section{Acknowledgements}

S. Gamba thanks all contributing authors for their courtesy and advice. D.P. Modest received a research grant from the University of Munich for this project (FöFoLe number 745) and thanks Professor Dr W. Hiddemann and Professor Dr H. Ostermann for their support. This work was further supported by a grant from the Deutsche Forschungsgemeinschaft DFG BR 1614/7-1 to C.J. Bruns and the DKTK/DKFZ 2013 ('Stem cells in Oncology') to C.J. Bruns. Horizon Discovery kindly supported this investigation by providing isogenic SW48-based cell lines on an academic research basis. Boehringer Ingelheim provided afatinib for this project.

\section{Conflicts of interest}

V. Heinemann received a research grant from Boehringer Ingelheim for a clinical trial. For the remaining authors there are no conflicts of interest.

\section{References}

1 Ciardiello F, Tortora G. EGFR antagonists in cancer treatment. N Engl J Med 2008; 358:1160-1174.

2 Fiske WH, Threadgill D, Coffey RJ. ERBBs in the gastrointestinal tract: recent progress and new perspectives. Exp Cell Res 2009; 315:583-601.

3 Scaltriti M, Baselga J. The epidermal growth factor receptor pathway: a model for targeted therapy. Clin Cancer Res 2006; 12:5268-5272.

4 Yarden Y, Sliwkowski MX. Untangling the ErbB signalling network. Nat Rev Mol Cell Biol 2001; 2:127-137.

5 Saltz LB, Meropol NJ, Loehrer PJ Sr, Needle MN, Kopit J, Mayer RJ. Phase II trial of cetuximab in patients with refractory colorectal cancer that expresses the epidermal growth factor receptor. J Clin Oncol 2004; 22:1201-1208.

6 Chung KY, Shia J, Kemeny NE, Shah M, Schwartz GK, Tse A, et al. Cetuximab shows activity in colorectal cancer patients with tumors that do not express the epidermal growth factor receptor by immunohistochemistry. J Clin Oncol 2005; 23:1803-1810.

7 Cunningham D, Humblet $\mathrm{Y}$, Siena $\mathrm{S}$, Khayat $\mathrm{D}$, Bleiberg $\mathrm{H}$, Santoro A, et al. Cetuximab monotherapy and cetuximab plus irinotecan in irinotecanrefractory metastatic colorectal cancer. N Engl J Med 2004; 351:337-345.

8 Jonker DJ, O'Callaghan CJ, Karapetis CS, Zalcberg JR, Tu D, Au HJ, et al. Cetuximab for the treatment of colorectal cancer. N Engl J Med 2007; 357:2040-2048.

9 Van Cutsem E, Peeters M, Siena S, Humblet Y, Hendlisz A, Neyns B, et al. Open-label phase III trial of panitumumab plus best supportive care compared with best supportive care alone in patients with chemotherapyrefractory metastatic colorectal cancer. J Clin Oncol 2007; 25:1658-1664.

10 Benvenuti S, Sartore-Bianchi A, Di Nicolantonio F, Zanon C, Moroni M, Veronese $\mathrm{S}$, et al. Oncogenic activation of the RAS/RAF signaling pathway impairs the response of metastatic colorectal cancers to anti-epidermal growth factor receptor antibody therapies. Cancer Res 2007; 67:2643-2648.

11 De Roock W, Piessevaux H, De Schutter J, Janssens M, De Hertogh G, Personeni $\mathrm{N}$, et al. KRAS wild-type state predicts survival and is associated to early radiological response in metastatic colorectal cancer treated with cetuximab. Ann Oncol 2008; 19:508-515.

12 Karapetis CS, Khambata-Ford S, Jonker DJ, O'Callaghan CJ, Tu D, Tebbutt NC, et al. K-ras mutations and benefit from cetuximab in advanced colorectal cancer. N Engl J Med 2008; 359:1757-1765.

13 Lièvre A, Bachet JB, Boige V, Cayre A, Le Corre D, Buc E, et al. KRAS mutations as an independent prognostic factor in patients with advanced colorectal cancer treated with cetuximab. J Clin Oncol 2008; 26:374-379.

14 Amado RG, Wolf M, Peeters M, Van Cutsem E, Siena S, Freeman DJ, et al. Wild-type KRAS is required for panitumumab efficacy in patients with metastatic colorectal cancer. J Clin Oncol 2008; 26:1626-1634.

15 Spano JP, Milano G, Vignot S, Khayat D. Potential predictive markers of response to EGFR-targeted therapies in colorectal cancer. Crit Rev Oncol Hematol 2008; 66:21-30.

16 Peeters M, Douillard JY, Van Cutsem E, Siena S, Zhang K, Williams R, Wiezorek J. Mutant KRAS codon 12 and 13 alleles in patients with metastatic colorectal cancer: assessment as prognostic and predictive biomarkers of response to panitumumab. J Clin Oncol 2013; 31:759-765.

17 Bos JL, Fearon ER, HamiltonSR, Verlaan-de Vries M, van Boom JH, van der $\mathrm{Eb} \mathrm{AJ}$, Vogelstein B. Prevalence of ras gene mutations in human colorectal cancers. Nature 1987; 327:293-297.

18 Schubbert S, Shannon K, Bollag G. Hyperactive Ras in developmental disorders and cancer. Nat Rev Cancer 2007; 7:295-308.

19 Bardelli A, Siena S. Molecular mechanisms of resistance to cetuximab and panitumumab in colorectal cancer. J Clin Oncol 2010; 28:1254-1261.

20 Samowitz WS, Curtin K, Schaffer D, Robertson M, Leppert M, Slattery ML. Relationship of Ki-ras mutations in colon cancers to tumor location, stage, and survival: a population-based study. Cancer Epidemiol Biomarkers Prev 2000; 9:1193-1197.

21 De Roock W, Jonker DJ, Di Nicolantonio F, Sartore-Bianchi A, Tu D, Siena S, et al. Association of KRAS p.G13D mutation with outcome in patients with chemotherapy-refractory metastatic colorectal cancer treated with cetuximab. JAMA 2010; 304:1812-1820.

22 Tejpar S, Celik I, Schlichting M, Sartorius U, Bokemeyer C, Van Cutsem E. Association of KRAS G13D tumor mutations with outcome in patients with metastatic colorectal cancer treated with first-line chemotherapy with or without cetuximab. J Clin Oncol 2012; 30:3570-3577.

23 Sharma PS, Sharma R, Tyagi T. Receptor tyrosine kinase inhibitors as potent weapons in war against cancers. Curr Pharm Des 2009; 15:758-776.

24 Lynch TJ, Bell DW, Sordella R, Gurubhagavatula S, Okimoto RA, Brannigan BW, et al. Activating mutations in the epidermal growth factor receptor underlying responsiveness of non-small-cell lung cancer to gefitinib. N Engl J Med 2004; 350:2129-2139.

25 Paez JG, Jänne PA, Lee JC, Tracy S, Greulich H, Gabriel S, et al. EGFR mutations in lung cancer: correlation with clinical response to gefitinib therapy. Science 2004; 304:1497-1500.

26 Pao W, Miller V, Zakowski M, Doherty J, Politi K, Sarkaria I, et al. EGF receptor gene mutations are common in lung cancers from 'never smokers' and are associated with sensitivity of tumors to gefitinib and erlotinib. Proc Natl Acad Sci USA 2004; 101:13306-13311.

27 Li D, Ambrogio L, Shimamura T, Kubo S, Takahashi M, Chirieac LR, et al. BIBW2992, an irreversible EGFR/HER2 inhibitor highly effective in preclinical lung cancer models. Oncogene 2008; 27:4702-4711.

28 Yang JC, Shih JY, Su WC, Hsia TC, Tsai CM, Ou SH, et al. Afatinib for patients with lung adenocarcinoma and epidermal growth factor receptor mutations (LUX-Lung 2): a phase 2 trial. Lancet Oncol 2012; 13:539-548.

29 Lin NU, Winer EP, Wheatley D, Carey LA, Houston S, Mendelson D, et al. A phase Il study of afatinib (BIBW 2992), an irreversible ErbB family blocker, in patients with HER2-positive metastatic breast cancer progressing after trastuzumab. Breast Cancer Res Treat 2012; 133:1057-1065.

30 Heinemann V. Afatinib as Cancer Therapy for Exocrine Pancreatic Tumours (ACCEPT). Identifier: NCT01728818. Bethesda, Maryland: US National Library of Medicine, 2000 [cited 30 November 2014]. Available at: $h t t p: / /$ clinicaltrials.gov/show/NCT01728818 NLM.

31 Britten CD. Targeting ErbB receptor signaling: a pan-ErbB approach to cancer. Mol Cancer Ther 2004; 3:1335-1342. 
32 Grandis JR, Sok JC. Signaling through the epidermal growth factor receptor during the development of malignancy. Pharmacol Ther 2004; 102:37-46.

33 Citri A, Skaria KB, Yarden Y. The deaf and the dumb: the biology of ErbB-2 and ErbB-3. Exp Cell Res 2003; 284:54-65.

34 Olayioye MA, Neve RM, Lane HA, Hynes NE. The ErbB signaling network: receptor heterodimerization in development and cancer. EMBO J 2000; 19:3159-3167.

35 McCormick F. Success and failure on the ras pathway. Cancer Biol Ther 2007; 6:1654-1659.

36 Rodriguez-Viciana P, Warne PH, Dhand R, Vanhaesebroeck B, Gout I, Fry MJ, et al. Phosphatidylinositol-3-OH kinase as a direct target of Ras. Nature $1994 ; 370: 527-532$.

37 Zhang XF, Settleman J, Kyriakis JM, Takeuchi-Suzuki E, Elledge SJ, Marshall MS, et al. Normal and oncogenic p21ras proteins bind to the aminoterminal regulatory domain of c-Raf-1. Nature 1993; 364:308-313.

38 Downward J. Targeting RAS signalling pathways in cancer therapy. Nat Rev Cancer 2003; 3:11-22.

39 Der CJ, Van Dyke T. Stopping ras in its tracks. Cell 2007; 129:855-857

40 Pao W, Wang TY, Riely GJ, Miller VA, Pan Q, Ladanyi M, et al. KRAS mutations and primary resistance of lung adenocarcinomas to gefitinib or erlotinib. PLoS Med 2005; 2:e17.

41 Di Nicolantonio F, Arena S, Gallicchio M, Zecchin D, Martini M, Flonta SE, et al. Replacement of normal with mutant alleles in the genome of normal human cells unveils mutation-specific drug responses. Proc Natl Acad Sci USA 2008; 105:20864-20869.

42 Khan IF, Hirata RK, Russell DW. AAV-mediated gene targeting methods for human cells. Nat Protoc 2011; 6:482-501.

43 Konishi H, Karakas B, Abukhdeir AM, Lauring J, Gustin JP, Garay JP, et al. Knock-in of mutant K-ras in nontumorigenic human epithelial cells as a new model for studying K-ras mediated transformation. Cancer Res 2007; 67:8460-8467.
44 Neumann J, Zeindl-Eberhart E, Kirchner T, Jung A. Frequency and type of KRAS mutations in routine diagnostic analysis of metastatic colorectal cancer. Pathol Res Pract 2009; 205:858-862.

45 Bazan V, Migliavacca M, Zanna I, Tubiolo C, Grassi N, Latteri MA, et al Specific codon $13 \mathrm{~K}$-ras mutations are predictive of clinical outcome in colorectal cancer patients, whereas codon $12 \mathrm{~K}$-ras mutations are associated with mucinous histotype. Ann Oncol 2002; 13:1438-1446.

46 Hamada K, Monnai M, Kawai K, Nishime C, Kito C, Miyazaki N, et al. Liver metastasis models of colon cancer for evaluation of drug efficacy using NOD/Shi-scid IL2Rgammanull (NOG) mice. Int J Onco/ 2008; 32:153-159.

47 Yonesaka K, Zejnullahu K, Okamoto I, Satoh T, Cappuzzo F, Souglakos J, et al. Activation of ERBB2 signaling causes resistance to the EGFR-directed therapeutic antibody cetuximab. Sci Transl Med 2011; 3:99ra86.

48 Erjala K, Sundvall M, Junttila TT, Zhang N, Savisalo M, Mali P, et al. Signaling via ErbB2 and ErbB3 associates with resistance and epidermal growth factor receptor (EGFR) amplification with sensitivity to EGFR inhibitor gefitinib in head and neck squamous cell carcinoma cells. Clin Cancer Res 2006; 12:4103-4111.

49 Dimou A, Syrigos K, Saif MW. Novel modalities in the treatment of patients with KRAS-mutated colorectal cancer. Anticancer Drugs 2011; 22:384-391.

50 Modest DP, Camaj P, Heinemann V, Schwarz B, Jung A, Laubender RP, et al. KRAS allele-specific activity of sunitinib in an isogenic disease model of colorectal cancer. J Cancer Res Clin Oncol 2013; 139:953-961.

51 Ebi H, Corcoran RB, Singh A, Chen Z, Song Y, Lifshits E, et al. Receptor tyrosine kinases exert dominant control over PI3K signaling in human KRAS mutant colorectal cancers. J Clin Invest 2011; 121:4311-4321.

52 Modest DP, Brodowicz T, Stintzing S, Jung A, Neumann J, Laubender RP, et al. Impact of the specific mutation in KRAS codon 12 mutated tumors on treatment efficacy in patients with metastatic colorectal cancer receiving cetuximab-based first-line therapy: a pooled analysis of three trials. Oncology 2012; 83:241-247. 